\title{
LV-OP-2-1
}

\section{Prediction of microvascular invasion via gadoxetic acid-enhanced MRI in hepatocellular carcinoma: The implication of planning extent of the hepatectomy}

\author{
Hyeo Seong HWANG ${ }^{1}$, Heejin BAE ${ }^{2}$, Mi-Suk PARK ${ }^{2}$, Dai Hoon $\mathrm{HAN}^{3}$, Gi Hong $\mathrm{CHOI}^{* 3}$, Kyung Sik KIM ${ }^{3}$, Jin Sub $\mathrm{CHOI}^{3}$
}

'National Health Insurance Service Ilsan Hospital, Goyang, Korea

${ }^{2}$ Department of Radiology and Research Institute of Radiological Science, Severance Hospital, Yonsei University College of Medicine, Seoul, Korea ${ }^{3}$ Department of Surgery, Yonsei University College of Medicine, Seoul, Korea

Introduction: The purpose of this study was to identify predictive factors for microvascular invasion (MVI) in hepatocellular carcinoma (HCC) using clinical factors and imaging findings of magnetic resonance imaging (MRI), to divide the patients into a subgroup with similar prognosis and finally to evaluate whether the extent of hepatectomy can affect long-term outcomes in the subgroup.

Methods: A total of 410 patients with surgically resected single HCC $(\leq 5 \mathrm{~cm})$ who underwent preoperative gadoxetic acid-enhanced MRI were included. Significant predictive factors for MVI were identified via univariate and multivariate analysis and used to divide the patients into low and high-risk groups. In the subgroup, long-term outcomes were analyzed after the minor versus major hepatectomy.

Results: Four variables were independently associated with MVI: alpha-fetoprotein $\geq 400 \mathrm{ng} / \mathrm{mL}(p=0.021)$, tumor size $\geq 3 \mathrm{~cm}(p<$ $0.001)$, non-smooth tumor margin $(p<0.001)$ and arterial peritumoral enhancement $(p=0.001)$. Patients with a combination of three or more factors were classified as the high-risk group $(\mathrm{n}=103)$, which showed worse prognosis than the low-risk group ( $\mathrm{n}=307)(p<$ 0.001). While the extent of hepatectomy was not associated with prognosis in the low-risk group, major hepatectomy significantly improved disease-free survival $(p<0.011)$ and decreased early intrahepatic recurrence $(<2$ years $)(p=0.005)$ than minor hepatectomy in the high-risk patients.

Conclusions: The patients who had a combination of three or more predictive factors for MVI showed worse prognosis after hepatectomy for HCC and major hepatectomy improved long-term outcomes in these high-risk patients. 